\title{
Three Ways to Solve for Bond Prices in the Vasicek Model
}

ROGEMAR S. MAMON $\dagger$

Department of Statistics, University of British Columbia

roge@stat.ubc.ca

Vancouver, BC, Canada V6T 1Z2

\begin{abstract}
Three approaches in obtaining the closed-form solution of the Vasicek bond pricing problem are discussed in this exposition. A derivation based solely on the distribution of the short rate process is reviewed. Solving the bond price partial differential equation (PDE) is another method. In this paper, this PDE is derived via a martingale approach and the bond price is determined by integrating ordinary differential equations. The bond pricing problem is further considered within the Heath-Jarrow-Morton (HJM) framework in which the analytic solution follows directly from the short rate dynamics under the forward measure.
\end{abstract}

Keywords: Bond pricing, Vasicek model, Martingales, HJM methodology, Forward measure.

\section{Introduction}

Vasicek's pioneering work (1977) is the first account of a bond pricing model that incorporates stochastic interest rate. The short rate dynamics is modeled as a diffusion process with constant parameters. When the bond price is based on this assumption, it has the feature that on a given date, the ratio of expected excess return per unit of volatility (the market price of risk) is the same, regardless of bond's maturity. Vasicek's model is a special version of Ornstein-Uhlenbeck $(\mathrm{O}-\mathrm{U})$ process, with constant volatility. This implies that the short rate is both Gaussian and Markovian. The model also exhibits mean-reversion and is therefore able to capture monetary authority's behavior of setting target rates. Furthermore, historical experience of interest rates justifies the O-U specification.

Given the pedagogical value of the Vasicek model in stochastic term structure modeling, the purpose of this paper is to present alternative derivation of the bond price solution. From the bond price the entire yield curve can be constructed at any given time. Thus, in turn, the term structure dynamics is characterized by the evolution of the short rate.

$\dagger$ Requests for reprints should be sent to Rogemar S. Mamon, Department of Statistics, University of British Columbia, Vancouver, BC, Canada V6T 1 Z2. 
Vasicek model's tractability property in bond pricing and the model's interesting stochastic characteristics make this classical model quite popular. In this paper a review of short rate's stochastic properties relevant to the derivation of the closed-form solution of the bond price within the Vasicek framework is presented. These properties become the basis for the first method examined in section 2. Under this technique, the bond price is derived from the implications of the interest rate's probability distribution. The development of the theory under this set-up follows from the outline of Lamberton and Lapeyre (1995).

The orginal derivation of the explicit formula for the bond price was based on solving the PDE that must be satisfied by the bond price. This is done by constructing a locally riskless portfolio and using the no-arbitrage arguments. Duffie and Kan (1996) provide a further characterization of this PDE. They prove that, if some Ricatti equations have solutions to the required maturity, the bond price has an exponential affine form. Vasicek's model belongs to this exponential affine class because the specification of its drift and volatility gives rise to a solvable set of equations in accordance with the Duffie-Kan descriptions. The second approach discussed in section 3 relies on the solution of the bond price PDE. However, unlike the traditional approach, this paper presents a martingale-oriented derivation of this PDE. This is motivated by the equivalence of the no-arbitrage pricing technique and the risk-neutral valuation which is a martingale-based method. Recently, Elliott and Van der Hoek (2001) offer a new method of solving the problem studied by Duffie and Kan. In their paper, it is shown that, when the short rate process is given by Gaussian dynamics or square root processes, the bond price is an exponential affine function. Their technique determines the bond price by integrating linear ODE and Ricatti equations are not needed. A similar idea is applied here to provide a solution to the bond pricing problem in the Vasicek model.

Section 4 presents a third alternative that considers the Heath-JarrowMorton (HJM) pricing paradigm. The equivalence between the forward rate and the conditional expectation of the short rate under the forward measure is discussed. Elaborating on the work of Geman, El Karoui and Rochet (1995) using the bond price as a numéraire, the short rate's dynamics is obtained under the forward measure. Consequently, the Vasicek forward rate dynamics is explicitly determined and therefore the analytic bond price follows immediately from the HJM bond pricing formula. 


\section{Bond Price Implied by the Short Rate Distribution}

In modeling the uncertainty of interest rates, assume that there is an underlying probability space $(\Omega, \mathcal{F}, P)$ equipped with a standard filtration $\left\{\mathcal{F}_{t}\right\}$. Under the risk-neutral measure $P$, the short rate dynamics is given by

$$
d r_{t}=a\left(b-r_{t}\right) d t+\sigma d W_{t}
$$

where $a, b$ and $\sigma$ are all positive constants.

It can be verified using Itô's formula that

$$
r_{t}=e^{-a t}\left[r_{0}+\int_{0}^{t} a b e^{a u} d u+\sigma \int_{0}^{t} e^{a u} d W_{u}\right]
$$

is a solution to the stochastic differential equation (SDE) in (1). Note further that

$$
\begin{aligned}
r_{t} & =e^{-a t}\left[r_{0}+b\left(e^{a t}-1\right)+\int_{0}^{t} \sigma e^{a u} d W_{u}\right] \\
& =\mu_{t}+\sigma \int_{0}^{t} e^{a(u-t)} d W_{u},
\end{aligned}
$$

where $\mu_{t}$ is a deterministic function. Clearly, $E\left[r_{t}\right]=\mu_{t}$.

Observe further that $r_{t}$ is a Gaussian random variable. This follows from the definition of the stochastic integral term, which is $\lim _{|\pi| \rightarrow 0} \sum_{i=0}^{n-1} e^{a\left(u_{i}-t\right)}\left(W_{u_{i+1}}-\right.$ $\left.W_{u_{i}}\right)$ and the increment $\left(W_{u_{i+1}}-W_{u_{i}}\right) \sim N\left(0, u_{i+1}-u_{i}\right)$. In general, if $\delta$ is deterministic (i.e., a function only of $t$ ), $\int_{0}^{t} \delta(u) d W_{u}$ is Gaussian.

While the expectation follows immediately from the solution for $r_{t}$ given above, $E\left[r_{t}\right]$ can be determined without necessarily solving explicitly the SDE. Consider the integral form of (1). That is,

$$
r_{t}=r_{0}+\int_{0}^{t}\left(a\left(b-r_{u}\right) d u+\sigma d W_{u}\right) .
$$

Hence,

$$
\mu_{t}:=E\left[r_{t}\right]=r_{0}+\int_{0}^{t} a\left(b-E\left[r_{u}\right]\right) d u
$$

From (2),

$$
\frac{d}{d t} \mu_{t}=a\left(b-\mu_{t}\right)
$$

which is a linear ordinary differential equation (ODE). Consequently, using the integrating factor $e^{a t}$

$$
E\left[r_{t}\right]=e^{-a t}\left[r_{0}+b\left(e^{a t}-1\right)\right]=\mu_{t} .
$$


In this model, $b$ is some kind of level $r$ is trying to attain. We call this the mean-reverting level. Similarly, define

$$
\begin{aligned}
\sigma_{t}^{2}: & \left.=\operatorname{Var}\left[r_{t}\right]=E\left[\left(\sigma e^{-a t} \int_{0}^{t} e^{a u} d W_{u}\right)^{2}\right)\right] \\
& =\sigma^{2} e^{-2 a t} E\left[\int_{0}^{t} e^{2 a u} d u\right] \text { by Itô's isometry } \\
& =\sigma^{2}\left(\frac{1-e^{-2 a t}}{2 a}\right) .
\end{aligned}
$$

Therefore, $r_{t} \sim N\left(\mu_{t}, \sigma_{t}^{2}\right)$ with mean and variance given in (3) and (4), respectively.

Since normal random variables can become negative with positive probability, this is considered to be the weakness of the Vasicek model. Nevertheless, the simplicity and tractability of the model validate its discussion.

Using the risk-neutral valuation framework, the price of a zero-coupon bond with maturity $T$ at time $t$ is

$$
B(t, T)=E\left[\exp \left(-\int_{t}^{T} r_{u} d u\right) \mid \mathcal{F}_{t}\right] .
$$

Write

$$
X(u)=r_{u}-b .
$$

Here, $X(u)$ is the solution of the Ornstein-Uhlenbeck equation

$$
d X(t)=-a X(t)+\sigma d W_{t}
$$

with $X(0)=r_{0}-b$. Applying Itô's lemma, the $X(u)$ process is given by

$$
X(u)=e^{-a u}\left(X(0)+\int_{0}^{u} \sigma e^{a s} d W_{s}\right) .
$$

Clearly, $X(u)$ is a Gaussian process with continuous sample paths. If $X(u)$ is Gaussian then $\int_{0}^{t} X(u) d u$ is also Gaussian. Using (7), we obtain

$$
E[X(u)]=X(0) e^{-a u} .
$$

Thus,

$$
E\left[\int_{0}^{t} X(u) d u\right]=\frac{X(0)}{a}\left(1-e^{-a t}\right) .
$$


Similarly,

$$
\begin{aligned}
\operatorname{Cov}[X(t), X(u)] & =\sigma^{2} e^{-a(u+t)} E\left[\int_{0}^{t} e^{a s} d W_{s} \int_{0}^{u} e^{a s} d W_{s}\right] \\
& =\sigma^{2} e^{-a(u+t)} \int_{0}^{u \wedge t} e^{2 a s} d s=\frac{\sigma^{2}}{2 a} e^{-a(u+t)}\left(e^{2 a(u \wedge t)}-1\right) .
\end{aligned}
$$

Consequently,

$$
\begin{aligned}
& \operatorname{Var}\left[\int_{0}^{t} X(u) d u\right]=\operatorname{Cov}\left[\int_{0}^{t} X(u) d u, \int_{0}^{t} X(s) d s\right] \\
& =E\left[\left(\int_{0}^{t} X(u) d u-E\left[\int_{0}^{t} X(u) d u\right]\right)\left(\int_{0}^{t} X(s) d s-E\left[\int_{0}^{t} X(s) d s\right]\right)\right] \\
& =\int_{0}^{t} \int_{0}^{t} E[(X(u)-E[X(u)])(X(s)-E[X(s)])] d u d s \\
& =\int_{0}^{t} \int_{0}^{t} \operatorname{Cov}[X(u), X(s)] d u d s=\int_{0}^{t} \int_{0}^{t} \frac{\sigma^{2}}{2 a} e^{-a(u+s)}\left(e^{2 a(u \wedge s)}-1\right) d u d s \\
& =\frac{\sigma^{2}}{2 a^{3}}\left(2 a t-3+4 e^{-a t}-e^{-2 a t}\right) .
\end{aligned}
$$

From (5), we have

$$
E\left[-\int_{0}^{t} r_{u} d u\right]=E\left[-\int_{0}^{t}(X(u)+b) d u\right] .
$$

Therefore, together with equation (8)

$$
E\left[-\int_{t}^{T} r_{u} d u\right]=-\frac{r_{t}-b}{a}\left(1-e^{-a(T-t)}\right)-b(T-t) .
$$

Furthermore,

$$
\begin{aligned}
\operatorname{Var}\left[-\int_{t}^{T} r_{u} d u\right] & =\operatorname{Var}\left[\int_{t}^{T} X(u) d u\right] \\
& =\frac{\sigma^{2}}{2 a^{3}}\left(2 a(T-t)-3+4 e^{-a(T-t)}-e^{-2 a(T-t)(\jmath 1)}\right.
\end{aligned}
$$

by the result from (9).

From the Itô integral representation of $r_{t}$, we also note that the defining process for the short rate is also Markov. For proof, see Karatzas and Shreve, p. 355. 
Thus,

$$
B(t, T)=E\left[\exp \left(-\int_{t}^{T} r_{u} d u\right) \mid \mathcal{F}_{t}\right]=E\left[\exp \left(-\int_{t}^{T} r_{u} d u\right) \mid r_{t}\right] .
$$

We write

$B\left(t, T, r_{t}\right):=E\left[\exp \left(-\int_{t}^{T} r_{u} d u\right) \mid r_{t}\right]=E\left[\exp \left(-\int_{t}^{T} r_{u}\left(r_{t}\right) d u\right)\right]$.

That is, $r_{u}$ is a function of $r_{t}$.

Combining (10) and (11), the bond price is given by

$$
\begin{aligned}
B\left(t, T, r_{t}\right)=\exp \left(E\left[-\int_{t}^{T} r_{u}\left(r_{t}\right) d u\right]+\frac{1}{2} \operatorname{Var}\left[-\int_{t}^{T} r_{u}\left(r_{t}\right) d u\right]\right) \\
=\exp \left(-\frac{r_{t}-b}{a}\left(1-e^{-a(T-t)}\right)-b(T-t)\right. \\
\left.\quad+\frac{\sigma^{2}}{4 a^{3}}\left(2 a(T-t)-3+4 e^{-a(T-t)}-e^{-2 a(T-t)}\right)\right) \\
=\exp \left[-\left(\frac{1-e^{-a(T-t)}}{a}\right) r_{t}+b\left(\frac{1-e^{-a(T-t)}}{a}-(T-t)\right)\right. \\
\left.\quad-\frac{\sigma^{2}}{2 a^{2}}\left(\frac{1-e^{-a(T-t)}}{a}\right)+\frac{\sigma^{2}}{2 a^{2}}(T-t)-\frac{\sigma^{2}}{4 a}\left(\frac{1-2 e^{-a(T-t)}+e^{-2 a(T-t)}}{a^{2}}\right)\right] \\
=\exp \left[-A(t, T) r_{t}+b A(t, T)-b(T-t)-\frac{\sigma^{2}}{2 a^{2}} A(t, T)\right. \\
\left.+\frac{\sigma^{2}}{2 a^{2}}(T-t)-\frac{\sigma^{2}}{4 a} A(t, T)^{2}\right]=\exp \left(-A(t, T) r_{t}+D(t, T)\right),
\end{aligned}
$$

where

$$
\begin{aligned}
A(t, T) & =\frac{1-e^{-a(T-t)}}{a} \text { and } \\
D(t, T) & =\left(b-\frac{\sigma^{2}}{2 a^{2}}\right)[A(t, T)-(T-t)]-\frac{\sigma^{2} A(t, T)^{2}}{4 a}
\end{aligned}
$$

Since for all $t$, the yield $-\frac{\log B\left(t, T, r_{t}\right)}{T-t}$ obtained from (13) is affine in $r_{t}$, equation (13) is called an affine term structure model or an exponential affine bond price. 


\section{Solution via Bond Price PDE}

Under this approach, the derivation is based on the fact that the $r_{u}$ process is Markov. In other words, to determine how $r_{u}$ evolves from $t$ we need know only the value of $r_{t}, u \geq t$. Thus,

$$
B\left(t, T, r_{t}\right)=E\left[\exp \left(-\int_{t}^{T} r_{u}\left(r_{t}\right) d u\right) \mid r_{t}\right]
$$

and

$$
r_{u}=e^{-a(u-t)}\left[r_{t}+b\left(e^{a(u-t)}-1\right)+\sigma \int_{t}^{u} e^{a(v-t)} d W_{v}\right] .
$$

With $r_{t}$ as a parameter,

$$
\frac{\partial r_{u}\left(r_{t}\right)}{\partial r_{t}}=e^{-a(u-t)}
$$

So

$$
\int_{t}^{T} \frac{\partial r_{u}\left(r_{t}\right)}{\partial r_{t}} d u=\int_{t}^{T} e^{-a(u-t)} d u=\frac{1}{a}\left(1-e^{-a(T-t)}\right),
$$

which is deterministic.

Also,

$$
\begin{aligned}
\frac{\partial B\left(t, T, r_{t}\right)}{\partial r_{t}} & =E\left[-\left(\int_{t}^{T} \frac{\partial r_{u}\left(r_{t}\right)}{\partial r_{t}} d u\right) \exp \left(-\int_{t}^{T} r_{u}\left(r_{t}\right) d u\right)\right] \\
& =-\frac{1}{a}\left(1-e^{-a(T-t)}\right) E\left[\exp \left(-\int_{t}^{T} r_{u}\left(r_{t}\right) d u\right)\right] \\
& =-A(t, T) B\left(t, T, r_{t}\right),
\end{aligned}
$$

where $A(t, T)$ is given as in (14).

Thus, $\frac{\partial B}{\partial r_{t}}=-A B$. So,

$$
B\left(t, T, r_{t}\right)=C(t, T) \exp \left(-A(t, T) r_{t}\right),
$$

for some function $C$ independent of $r_{t}$.

Consider

$$
\exp \left(-\int_{0}^{t} r_{u}\left(r_{t}\right) d u\right) B\left(t, T, r_{t}\right)=E\left[\exp \left(-\int_{0}^{T} r_{u} d u\right) \mid \mathcal{F}_{t}\right] .
$$


Note that this is a $P$-martingale by the tower property. By Itô's lemma, we obtain

$$
\begin{aligned}
\exp & \left(-\int_{0}^{t} r_{u}\left(r_{t}\right) d u\right) B\left(t, T, r_{t}\right) \\
& =B\left(0, T, r_{0}\right)+\int_{0}^{t}-r_{u} \exp \left(-\int_{0}^{u} r_{v} d v\right) B\left(u, T, r_{u}\right) d u \\
& +\int_{0}^{t} \exp \left(-\int_{0}^{u} r_{v} d v\right) \frac{\partial}{\partial u} B\left(u, T, r_{u}\right) d u \\
& +\int_{0}^{t} \exp \left(-\int_{0}^{u} r_{v} d v\right) \frac{\partial}{\partial r_{u}} B\left(u, T, r_{u}\right)\left(a\left(b-r_{u}\right) d u+\sigma d W_{u}\right) \\
& +\frac{1}{2} \int_{0}^{t} \exp \left(-\int_{0}^{u} r_{v} d v\right) \frac{\partial^{2}}{\partial r_{u}^{2}} B\left(u, T, r_{u}\right) \sigma^{2} d u .
\end{aligned}
$$

Since this is a martingale, all the $d u$ terms must sum to zero. So,

$$
\begin{aligned}
-r_{t} B\left(t, T, r_{t}\right) & +\frac{\partial}{\partial t} B\left(t, T, r_{t}\right)+\frac{\partial}{\partial r_{t}} B\left(t, T, r_{t}\right)\left(a\left(b-r_{t}\right)\right) \\
& +\frac{\sigma^{2}}{2} \frac{\partial^{2}}{\partial r_{t}^{2}} B\left(t, T, r_{t}\right)=0 .
\end{aligned}
$$

Equation (16) is the PDE for the bond price in the Vasicek model. Moreover, this is a backward parabolic equation with $B\left(T, T, r_{t}\right)=1$ for every $r_{t}$.

So far we know

$$
B\left(t, T, r_{t}\right)=C(t, T) \exp \left(-A(t, T) r_{t}\right) .
$$

Therefore, we get the following partial derivatives.

$$
\begin{aligned}
\frac{\partial B}{\partial t} & =\frac{\partial C}{\partial t} \exp \left(-A(t, T) r_{t}\right)-C \frac{\partial A}{\partial t} r_{t} \exp \left(-A(t, T) r_{t}\right) \\
\frac{\partial B}{\partial r_{t}} & =-A C \exp \left(-A(t, T) r_{t}\right) \\
\frac{\partial^{2} B}{\partial r_{t}^{2}} & =A^{2} C \exp \left(-A(t, T) r_{t}\right)
\end{aligned}
$$

So, substituting to the PDE in (16) we have

$$
\begin{aligned}
& -r_{t} C \exp \left(-A r_{t}\right)+\frac{\partial C}{\partial t} \exp \left(-A r_{t}\right)-C \frac{\partial A}{\partial t} r_{t} \exp \left(-A r_{t}\right) \\
& -A C \exp \left(-A r_{t}\right)\left(a\left(b-r_{t}\right)\right)+\frac{\sigma^{2}}{2} A^{2} C \exp \left(-A r_{t}\right)=0 .
\end{aligned}
$$


Therefore,

$$
-r_{t} C+\frac{\partial C}{\partial t}-C \frac{\partial A}{\partial t} r_{t}-A C\left(a\left(b-r_{t}\right)\right)+\frac{\sigma^{2}}{2} A^{2} C=0 .
$$

Now, $B(t, T, 0)=C(t, T)$ and by putting $r_{t}=0$ we get

$$
\frac{\partial C}{\partial t}-a b A C+\frac{\sigma^{2}}{2} A^{2} C=0
$$

Noting again that we are solving a backward ODE with $C(T, T)=1$, we get

$$
\begin{aligned}
C(t, T)= & \exp \left[-\frac{a b}{a} \int_{t}^{T}\left(1-e^{-a(T-u)}\right) d u+\frac{\sigma^{2}}{2 a^{2}} \int_{t}^{T}\left(1-e^{-a(T-u)}\right)^{2} d u\right] \\
= & \exp \left[-b(T-t)+\frac{b}{a}\left(1-e^{-a(T-t)}\right)+\frac{\sigma^{2}}{2 a^{2}}(T-t)\right. \\
& \left.+\frac{\sigma^{2}}{4 a^{3}}\left(1-e^{-2 a(T-t)}\right)-\frac{\sigma^{2}}{a^{3}}\left(1-e^{-a(T-t)}\right)\right]
\end{aligned}
$$

Write

$$
D(t, T):=\log C(t, T) .
$$

We see that this reconciles with the second to the last terms of equation (12) and hence with the expression of equation (15). Under this approach, we have

$$
B\left(t, T, r_{t}\right)=\exp \left(-A(t, T) r_{t}+D(t, T)\right)
$$

where $A(t, T)$ is given by (14).

\section{Bond Pricing by HJM Methodology}

Following the terminology and notation of Heath, Jarrow and Morton (1992), this pricing paradigm is based on the concept of forward rate. The instantaneous forward rate at time $t$ for date $T>t$ is defined by

$$
f(t, T)=-\left.\frac{\partial \log B\left(t, t^{\prime}\right)}{\partial t^{\prime}}\right|_{t^{\prime}=T>t} .
$$

This refers to the rate of interest that must be paid between $t^{\prime}$ and $T$. It is known at time $t$ and therefore $\mathcal{F}_{t}$-measurable. Solving the differential equation in (17), yields

$$
B(t, T)=\exp \left(-\int_{t}^{T} f(t, u) d u\right) .
$$


The short rate at time $t, r_{t}$, is the instantaneous rate at time $t$, i.e., $r_{t}=f(t, t)$ for every $t \in[0, T]$. From equation (18), it is clear that once $f(t, T)$ is completely determined the bond price immediately follows. The dynamics of the forward rate and that of the short rate are related via the forward measure. Invoking the insights of Geman, El Karoui and Rochet (1995), the forward measure $P^{T}$ is defined on $\mathcal{F}_{T}$ by setting

$$
\left.\frac{d P^{T}}{d P}\right|_{\mathcal{F}_{T}}=\Lambda_{T}:=\frac{\exp \left(-\int_{0}^{T} r_{u} d u\right)}{B\left(0, T, r_{0}\right)} .
$$

Consider the Radon-Nikodým process

$$
\Lambda_{t}:=E\left[\Lambda_{T} \mid \mathcal{F}_{t}\right]:=\frac{\exp \left(-\int_{0}^{t} r_{u} d u\right) B(t, T)}{B(0, T)}, \quad t \in[0, T] .
$$

For any $\mathcal{F}_{T}$-measurable random variable $X$ we have

$$
\begin{aligned}
E^{T}\left[X \mid \mathcal{F}_{t}\right] & =\Lambda_{t}{ }^{-1} E\left[X \cdot \Lambda_{T} \mid \mathcal{F}_{t}\right] \\
& =E\left[\frac{X \exp \left(-\int_{t}^{T} r_{u} d u\right)}{B(t, T)} \mid \mathcal{F}_{t}\right] .
\end{aligned}
$$

Now, the bond price in terms of the short rate is given by

$$
B(t, T)=E\left[\exp \left(-\int_{t}^{T} r_{u} d u\right) \mid \mathcal{F}_{t}\right] .
$$

Differentiating with respect to $T$, we get

$$
\frac{\partial B(t, T)}{\partial T}=E\left[-r_{T} \exp \left(-\int_{t}^{T} r_{u} d u\right) \mid \mathcal{F}_{t}\right]=-E^{T}\left[r_{T} \mid \mathcal{F}_{t}\right] B(t, T),
$$

where the last equality follows from (19) with $X=r_{T}$. The bond price in terms of the forward rate is given in equation (18). Thus, differentiating $B(t, T)$ with respect to $T$, we obtain

$$
\frac{\partial B(t, T)}{\partial T}=-B(t, T) f(t, T) .
$$

Comparing (20) and (21), in terms of the short rate model, the forward rate is given by

$$
f(t, T)=E^{T}\left[r_{T} \mid \mathcal{F}_{t}\right]
$$

where $E^{T}$ denotes the expectation under $P^{T}$. 
Invoking the change of probability measures and numéraire technique, under the forward measure $P^{T}$, the stochastic dynamics for $r_{t}$ is given by

$$
d r_{t}=\left(a b-A(t, T) \sigma^{2}-a r_{t}\right) d t+\sigma d W_{t}^{T},
$$

where $W_{t}^{T}$ is the $P^{T}-$ Brownian motion defined by

$$
d W_{t}^{T}=d W_{t}+\sigma A(t, T) d t
$$

and $A(t, T)$ is the function defined in equation (14). See Appendix for the proof of (23). By Itô's lemma, for $t \leq T$, the solution to (23) is given by

$$
\begin{aligned}
r_{T}=r_{t} e^{-a(T-t)} & +\left(b-\frac{\sigma^{2}}{a^{2}}\right)\left(1-e^{-b(T-t)}\right)+\frac{\sigma^{2}}{2 a^{2}}\left[e^{-a(T-t)}-e^{-2 a(T-t)}\right] \\
& +\sigma \int_{t}^{T} e^{-a(T-u)} d W_{u}^{T}
\end{aligned}
$$

Thus,

$$
\begin{aligned}
E^{u}\left[r_{u} \mid \mathcal{F}_{t}\right] & =r_{t} e^{-a(u-t)}+\left(b-\frac{\sigma^{2}}{2 a^{2}}\right)\left(1-e^{-a(u-t)}\right) \\
& +\frac{\sigma^{2}}{2 a^{2}}\left(e^{-a(u-t)}-e^{-2 a(u-t)}\right) .
\end{aligned}
$$

So,

$$
\begin{aligned}
\int_{t}^{T} E^{u}\left[r_{u} \mid \mathcal{F}_{t}\right] d u= & \frac{r_{t}}{a}\left[-e^{-a(u-t)}\right]_{t}^{T}+\left(b-\frac{\sigma^{2}}{2 a^{2}}\right)(T-t) \\
& -\left(b-\frac{\sigma^{2}}{2 a^{2}}\right)\left[-\frac{1}{a} e^{-a(u-t)}\right]_{t}^{T} \\
& +\frac{\sigma^{2}}{2 a^{3}}\left[-e^{-a(u-t)}\right]_{t}^{T}-\frac{\sigma^{2}}{4 a^{3}}\left[-e^{-2 a(u-t)}\right]_{t}^{T} \\
= & \frac{r_{t}}{a}\left(1-e^{-a(T-t)}\right)+\left(b-\frac{\sigma^{2}}{2 a^{2}}\right)(T-t) \\
& -\left(b-\frac{\sigma^{2}}{2 a^{2}}\right)\left(\frac{1-e^{-a(T-t)}}{a}\right)+\frac{\sigma^{2}}{2 a^{3}}\left(1-e^{-a(T-t)}\right) \\
& -\frac{\sigma^{2}}{4 a^{3}}\left(1-e^{-2 a(T-t)}\right) \\
= & r_{t} A(t, T)+\left(b-\frac{\sigma^{2}}{2 a^{3}}\right)[(T-t)+A(t, T)] \\
& +\sigma^{2}\left[\frac{1-e^{-a(T-t)}}{a}\right]^{2}
\end{aligned}
$$


Therefore,

$$
\begin{aligned}
B\left(t, T, r_{t}\right) & =\exp \left(-\int_{t}^{T} f(t, u) d u\right)=\exp \left(-\int_{t}^{T} E^{u}\left[r_{u} \mid \mathcal{F}_{t}\right] d u\right) \\
& =\exp \left(-r_{t} A(t, T)+D(t, T)\right)
\end{aligned}
$$

and the $A(t, T)$ and $D(t, T)$ values are in agreement with that of equations (14) and (15), respectively.

\section{Conclusion}

The pedagogical value of the Vasicek model is well-known in stochastic interest rate modeling. This paper contributes to the development of the available mathematical techniques in obtaining the closed-form solution of the bond price under the Vasicek framework. A discussion for each of the three different methods was provided. The first derivation considers the distributional properties of the short rate proces $r_{t}$. The simple Gaussian structure of $r_{t}$ leads to a closed-form solution of the bond price. The bond price backward PDE is also derived using a martingale-oriented methodology. This PDE together with the Vasicek dynamics is the basis of the second method which integrates ordinary differential equations to get the bond price. Turning to the HJM pricing framework, the third approach employs the dynamics of the forward rate to fully describe the bond price process. The forward rate is linked to the short rate via the forward measure. When the short rate dynamics is determined under the forward measure, the HJM bond price is obtained and this reconciles with the prices computed from the other two approaches.

\section{Acknowledgments}

The author wishes to thank an anonymous referee for many helpful suggestions.

\section{References}

1. D. Duffie and R. Kan. A Yield-Factor Model of Interest Rates. Mathematical Finance, 64: 379-406, 1996.

2. R.J. Elliott and J. van der Hoek. Stochastic flows and the forward measure. Finance and Stochastics, 5: 511-525, 2001. 
3. H. Geman, N. El Karoui and J. Rochet. Changes of Numéraire, Changes of Probability Measure and Option Pricing. Journal of Applied Probability, 32: 443-458, 1995.

4. D. Heath, R. Jarrow and A. Morton. Bond Pricing and the Term Structure of Interest Rates: A New Methodology. Econometrica, 60: 77105, 1992.

5. I. Karatzas and S. Shreve. Brownian Motion and Stochastic Calculus. Springer Verlag, Berlin-Heidelberg-New York, 1988.

6. D. Lamberton and B. Lapeyre. Introduction to Stochastic Calculus Applied to Finance. Chapman \& Hall, London, 1995.

7. O. Vasicek. An Equilibrium characterization of the Term Structure. Journal of Financial Economics, 5: 177-188, 1977.

\section{Appendix : Proof of Result in Equation 23}

Let $P$ be an equivalent martingale measure (EMM) for the numéraire $H_{t}$ and $Q$ an EMM for the numéraire $J_{t}$. Then for any $V_{T} \in L^{2}\left(\Omega, \mathcal{F}_{T}, P\right)$ and $V_{T} \in L^{2}\left(\Omega, \mathcal{F}_{T}, Q\right)$

$$
V_{t}:=E^{P}\left[V_{T} \frac{H_{t}}{H_{T}} \mid \mathcal{F}_{t}\right]=E^{Q}\left[V_{T} \frac{J_{t}}{J_{T}} \mid \mathcal{F}_{t}\right] .
$$

Assume that $P$ and $Q$ are equivalent and denote the Radon-Nikodým derivative of $Q$ with respect to $P$ by $\Gamma_{t}$. We then have

$$
E^{Q}\left[V_{T} \frac{J_{t}}{J_{T}} \mid \mathcal{F}_{t}\right]=E^{P}\left[V_{T} \frac{H_{t}}{H_{T}} \Gamma_{t} \mid \mathcal{F}_{t}\right] .
$$

In particular, $\Gamma_{t}=\frac{H_{T}}{H_{t}} \cdot \frac{J_{t}}{J_{T}}$ for $t<T$. Suppose that the process under some measure $P$ associated with numéraire $H_{t}$ is given by $d X_{t}=m\left(X_{t}, t\right) d t+$ $\sigma\left(X_{t}, t\right) d W_{t}$ for some functions $m\left(X_{t}, t\right)$ and $\sigma\left(X_{t}, t\right)$. We are interested on the process followed by $X_{t}$ under another measure $Q$ with numéraire $J_{t}$.

Consider $\Gamma_{t, T}=\frac{H_{T}}{H_{t}} \cdot \frac{J_{t}}{J_{T}}$. From Girsanov's theorem, if $W_{t}^{Q}$ is a Wiener process under $Q, W_{t}^{Q}=W_{t}^{P}-\int_{0}^{t} \theta_{u} d u$ where $d \Gamma_{t, T}=\Gamma_{t, T} \theta_{T} d W_{T}^{P}$ and $\theta_{t}$ can be determined. Moreover, conditional upon $\mathcal{F}_{t}, \Gamma_{t, T}$ is a process in $T$.

Let $H_{t}$ and $J_{t}$ have dynamics under $P$ given by

$$
d H_{t}=m_{H} d t+\sigma_{H} d W_{t}^{P} \text { and } d J_{t}=m_{J} d t+\sigma_{J} d W_{t}^{P} .
$$

Using these dynamics and noting that $\Gamma_{t}$ is a martingale under $P$, it can be verified that

$$
\frac{d \Gamma_{T}}{\Gamma_{T}}=\left(\frac{\sigma_{J}}{J_{T}}-\frac{\sigma_{H}}{H_{T}}\right) d W_{T}^{P} .
$$


So, $\theta_{t}=\frac{\sigma_{J}}{J_{t}}-\frac{\sigma_{H}}{H_{t}}$.

Applied to our current situation, suppose $P$ is the EMM under the bank account numéraire and $Q$ is the forward measure with bond as the associated numéraire. For $s<t<T$

$$
\Gamma_{t}:=\Gamma_{s, t}=\frac{J_{t}}{J_{s}} \cdot \frac{H_{s}}{H_{T}}=\frac{B(t, T)}{B(s, T)} \exp \left(-\int_{s}^{t} r_{u} d u\right) .
$$

Under measure $P, \frac{d B(t, T)}{B(t, T)}=r_{t} d t+\sigma_{B}(t) d W_{t}$ for some function $\sigma_{B}(t)$.

It is a straightforward calculation to show that the process $\Gamma_{t}=\Gamma_{s, t}$, conditional upon $\mathcal{F}_{s}$, satifies

$$
\frac{d \Gamma_{t}}{\Gamma_{t}}=\frac{d B(t, T)}{B(t, T)}-r_{t} d t=\sigma_{B}(t) d W_{t} .
$$

This implies that $W_{t}^{Q}=W_{t}^{P}-\int_{0}^{t} \sigma_{B}(u) d u$. Hence, if under $P$ we have the dynamics $d X_{t}=m\left(X_{t}, t\right) d t+\sigma\left(X_{t}, t\right) d W_{t}^{P}$ then the $Q$-process for $X_{t}$ is $d X_{t}=\left(m\left(X_{t}, t\right)+\sigma_{B}(t) \sigma\left(X_{t}, t\right)\right) d t+\sigma\left(X_{t}, t\right) d W_{t}^{Q}$.

Equation 23 follows from this result with $X=r, Q=P^{T}, \sigma\left(X_{t}, t\right)=\sigma$, $\sigma_{B}(t)=-A(t, T) \sigma$ and $m\left(X_{t}, t\right)=a\left(b-r_{t}\right)$. 


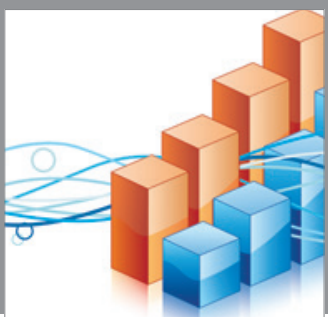

Advances in

Operations Research

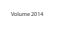

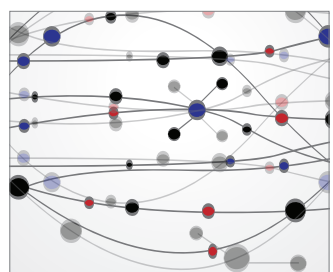

\section{The Scientific} World Journal
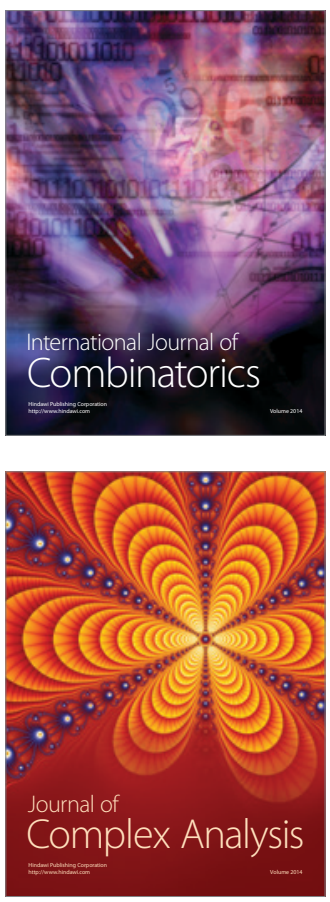

International Journal of

Mathematics and

Mathematical

Sciences
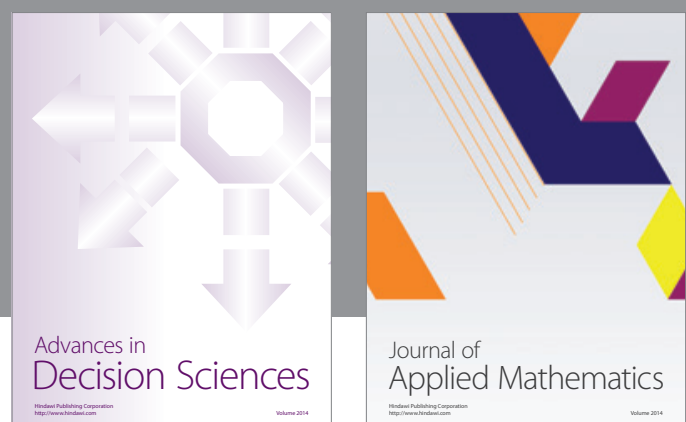

Journal of

Applied Mathematics
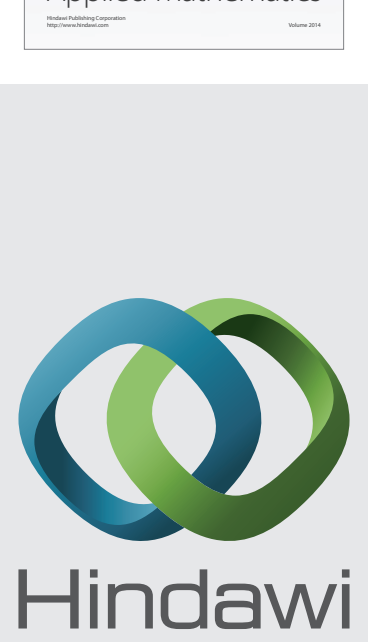

Submit your manuscripts at http://www.hindawi.com
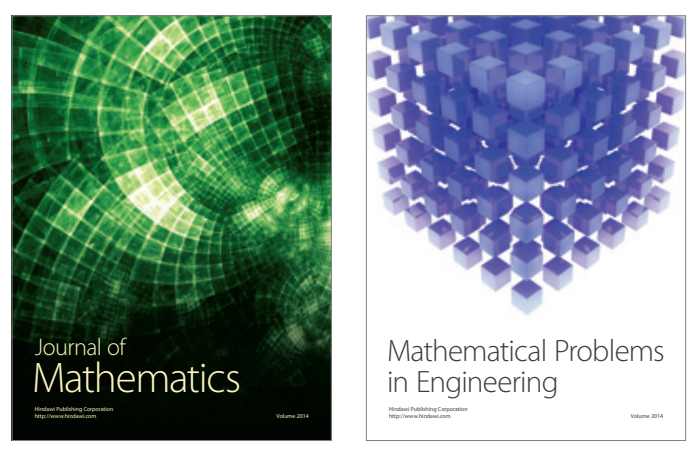

Mathematical Problems in Engineering
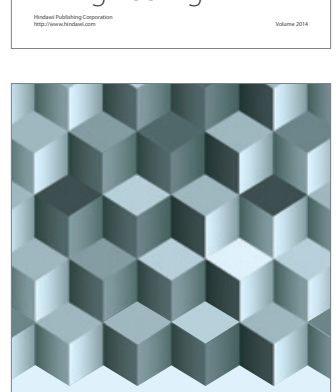

Journal of

Function Spaces
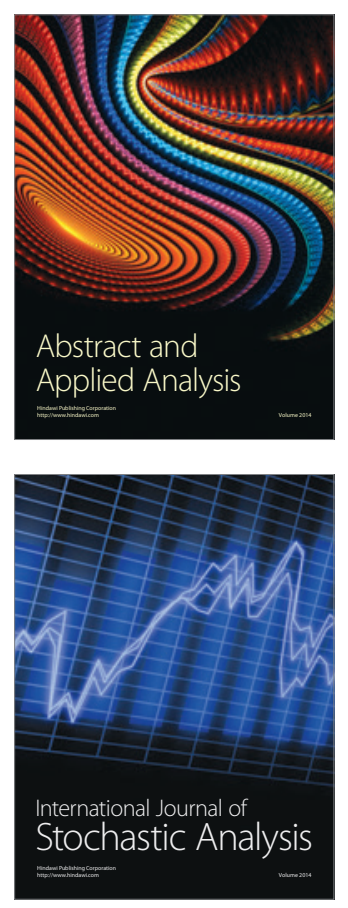

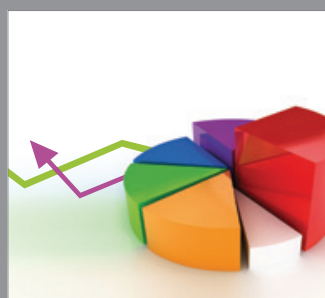

ournal of

Probability and Statistics

Promensencen
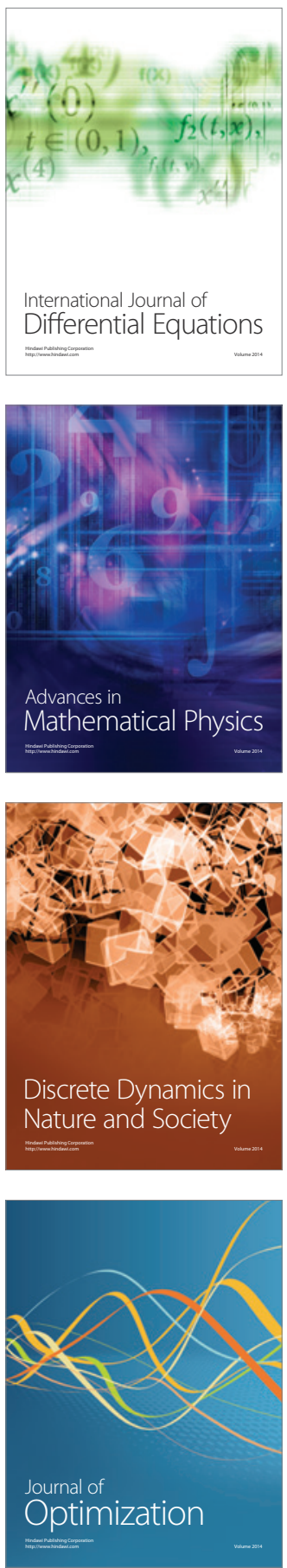